\title{
Orientation and Evolutionary Effects in Active Galactic Nuclei
}

\author{
Charlene A. Heisler \\ Mount Stromlo and Siding Spring Observatory, Institute of Advanced Studies, \\ Australian National University, Private Bag, Weston Creek PO, ACT 2611, Australia \\ heisler@mso.anu.edu.au \\ Received 1997 October 30, accepted 1998 April 15
}

\begin{abstract}
In this review I present observational results of active galactic nuclei that my colleagues and I have recently obtained. I discuss their significance in terms of unification schemes and evolutionary scenarios.
\end{abstract}

Keywords: galaxies: active — galaxies: Seyfert — galaxies: starburst — galaxies: jets — infrared: galaxies

\section{Introduction}

Active galactic nuclei (AGN) are thought to be powered by the accretion of gas onto a central massive black hole. The most common type of nearby AGN are Seyfert galaxies, named after Carl Seyfert (1943), who isolated and studied several galaxies with bright nuclei and relatively high-ionisation emission lines in their spectra, which were broader than the lower-ionisation emission lines observed in 'normal' galaxies. Seyfert galaxies are separated into two classes based on their emission line widths (e.g. Khachikian \& Weedman 1974; Osterbrock 1989). Seyfert 1 galaxies exhibit broad emission lines that are attributed to ionised gas within $1 \mathrm{pc}$ of the central massive black hole, whereas the spectra of Seyfert 2 galaxies only show narrower emission lines, believed to originate from a much larger region around the core. The standard model of AGN holds that a central engine (presumed to be accretion onto a super massive black hole) photoionises clouds within a parsec to produce broad lines observed in Seyfert 1 galaxies. Based on reverberation mapping, the spatial extent of the broad line emission is of the order of a few light-days to light-weeks (e.g. Peterson 1993), while narrow band imaging has clearly shown that the narrow line emission extends to $\sim$ kpc (e.g. Pogge 1989; Mulchaey, Wilson \& Tsvetanov 1996a, 1996b). The currently favoured paradigm for the unification of Seyfert galaxies, asserts that the central engine is surrounded by a dusty torus which obscures our view of the broad line region directly if viewed edge-on, resulting in the classification of a Seyfert 2 (e.g. Antonucci 1993; Urry \& Padovani 1995).

\section{Dusty Tori in Seyfert Galaxies}

The unified model for Seyfert galaxies critically depends upon the existence of a geometrically and optically thick dust torus surrounding the AGN core.
Theoretically it is very difficult to sustain a thick dusty torus in a galaxy, as it should either become a thin disk or dissipate over a very short period of time. But evidence has been accumulating which points to the existence of thick dusty tori in AGN. Indirect evidence for a thick torus is given by the numerous ionisation cones observed in Seyfert galaxies (e.g. Pogge 1989; Colbert et al. 1996; Simpson et al. 1997). Amongst the best direct evidence for a thick torus is the HST observation of NGC 4261, which clearly shows a large gaseous disk $\sim 250 \mathrm{pc}$ in diameter, and more recently, the direct observation of a parsec scale torus using high resolution radio continuum imaging (Gallimore, Baum \& O'Dea 1997).

\subsection{Spectropolarimetry}

2.1.1 Motivation. The detection of broad emission lines scattered into our line of sight by free electrons and/or dust in NGC 1068, the prototypical Seyfert 2 galaxy (Antonucci \& Miller 1985), and other Seyfert 2 galaxies (Young et al. 1996; Inglis et al. 1993; Hines \& Wills 1993; Tran, Miller \& Kay 1992; Miller \& Goodrich 1990) has strengthened the unified model for Seyfert galaxies. Unfortunately, all previous studies are known to be biased in their continuum polarisation properties, with samples chosen to be either highly polarised or having rising polarisation in the red. Such characteristics are not typical of Seyfert galaxies in general. In addition to these biases, there have been very few non-detections published, and there has been no consistency in ensuring non-detections are at the same signal-to-noise ratio in percentage polarised flux. To overcome these problems, Heisler, Lumsden \& Bailey (1997) undertook a new spectropolarimetry survey, based on a $60 \mu \mathrm{m}$ flux limited sample consisting of 16 Seyfert 2 galaxies. The $60 \mu \mathrm{m}$ emission is typically a measure of global emission from the galaxy, so is more isotropic than most properties that were previously used to define samples of AGN. 
2.1.2 What causes polarisation? There are several possible mechanisms that may contribute to polarisation in AGN.

(a) Non-thermal polarisation: Many high polarisation AGN (20-50\% polarised) display apparently nonthermal emission spectra and typically variable polarisation (e.g. blazars and powerful radio galaxies) which is attributed to synchrotron emission. According to the unification scenario, blazars are radio-loud AGN that contain a relativistic jet which is viewed along our line of sight (e.g. Urry \& Padovani 1995). Synchrotron emission occurs when high speed (close to velocity of light) charged particles rapidly revolve around a strong magnetic field, emitting highly plane-polarised electromagnetic waves with their polarisation plane perpendicular to the magnetic field. In blazars the synchrotron emission is believed to be produced in the relativistic jets.

(b) Thermal polarisation: Low polarisation AGN, such as Seyfert galaxies, are believed to be dominated by thermal polarisation processes.

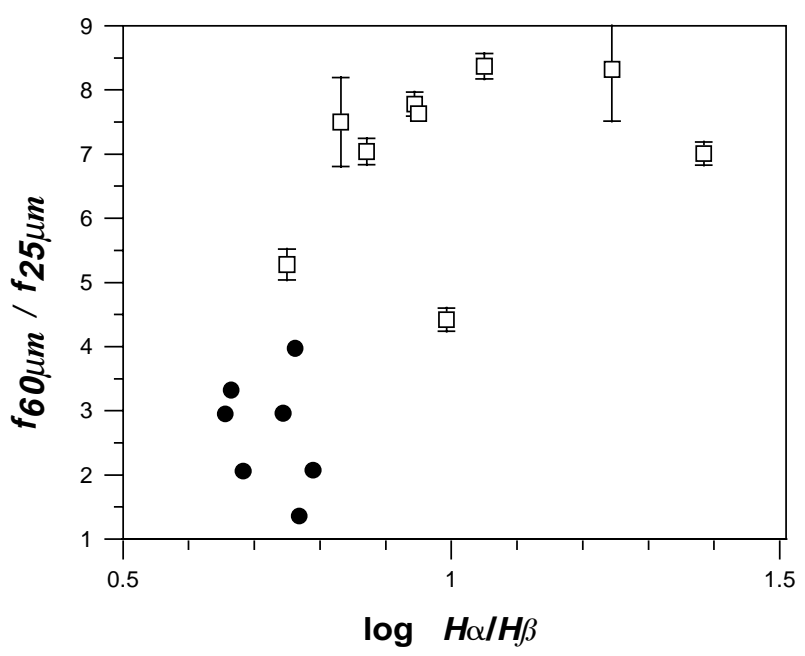

Figure 1 - Correlation between Balmer decrement $\log (\mathrm{H} \alpha / \mathrm{H} \beta)$, FIR flux ratio and the detectability of polarised broad lines in the IRAS-selected Seyfert 2 sample. The symbols have the following meaning: filled circles-polarised broad lines detected; open squares - polarised broad lines not detected. Optical spectropolarimetric observations were obtained at the Anglo-Australian Telescope during 16-18 August 1995. The half-waveplate module, built by the University of Hertfordshire, was used in conjunction with the RGO spectrograph, the $270 \mathrm{R}$ grating and the $25 \mathrm{~cm}$ camera, giving a spectral resolution of $8.4 \AA$. Our observations yield a three sigma detection at $0.5 \%$ polarisation.

(i) Scattering: Scattering of flux into the observers line of sight naturally produces polarisation. Scattering may be caused by dust grains or by electrons in AGN depending on where the scattering occurs. In the central regions of an AGN the gas that directly faces the AGN is highly ionised, and photons are scattered by free electrons, producing wavelength independent polarisation. In regions slightly further away from the central engine, dust scattering by circumnuclear molecular clouds may contribute to polarisation. The dependence of polarisation with wavelength for dust scattering depends on the scattering angle (Webb et al. 1993) and does not necessarily follow the simple Rayleigh scattering law in which the ionised flux rises towards shorter wavelengths.
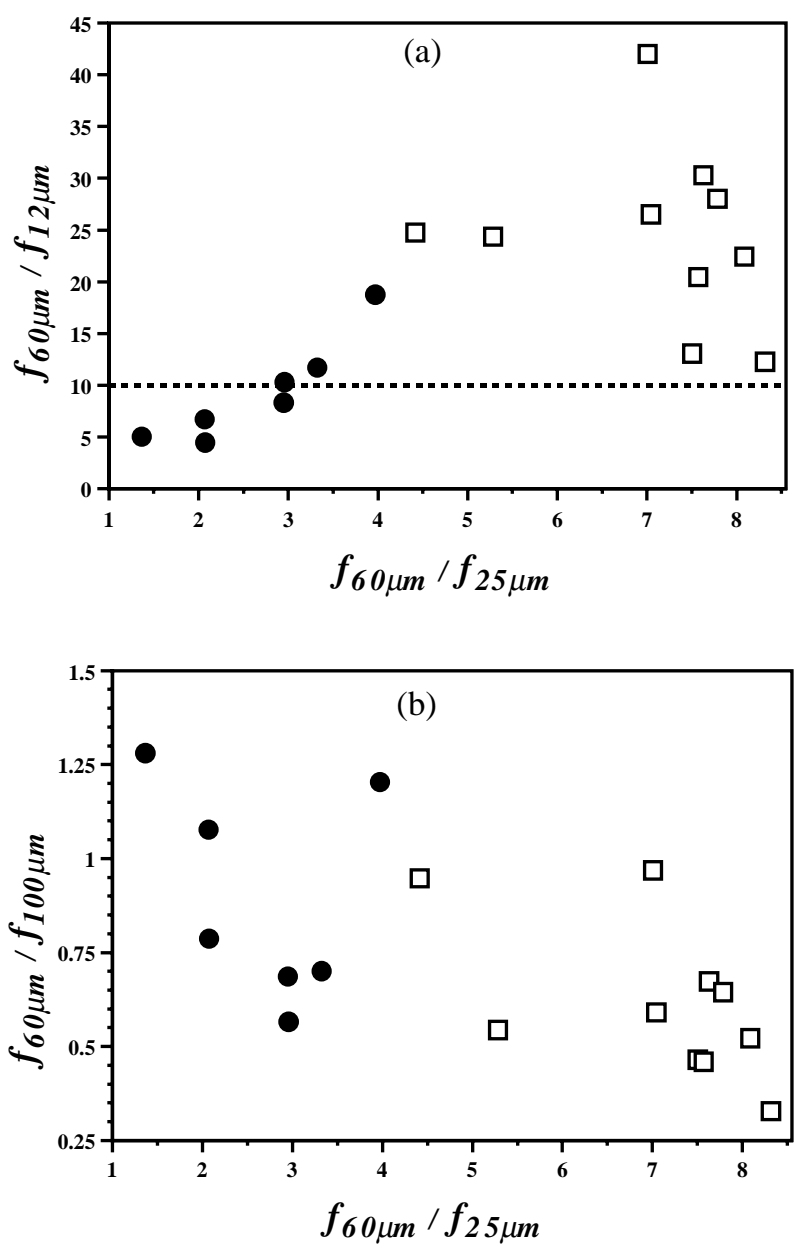

Figure 2-Far-infrared two-colour plots of (a) $f_{60} / f_{25}$ vs $f_{60} / f_{12}$ and (b) $f_{60} / f_{25}$ vs $f_{60} / f_{100}$, where the symbols have the same meaning as in Figure 1. The dashed line represents the boundary below which the majority of galaxies are classified as Seyfert 1 galaxies, based on the broad line region being directly visible in optical spectra (Spinoglio et al. 1995).

(ii) Dichroic extinction: Large scale alignment of nonspherical dust grains along the line of sight to the AGN can produce polarisation via dichroic extinction. Consider the simplified classical view of light where the electric and magnetic field vectors are perpendicular to each other and to the direction of propagation of the light. In the presence of a magnetic field, elongated dust grains will align their long axis perpendicular to the direction of the magnetic field. Light with electric vectors in the same direction as the elongated dust grains will be preferentially absorbed, producing a net polarisation which is parallel to the direction of the magnetic 


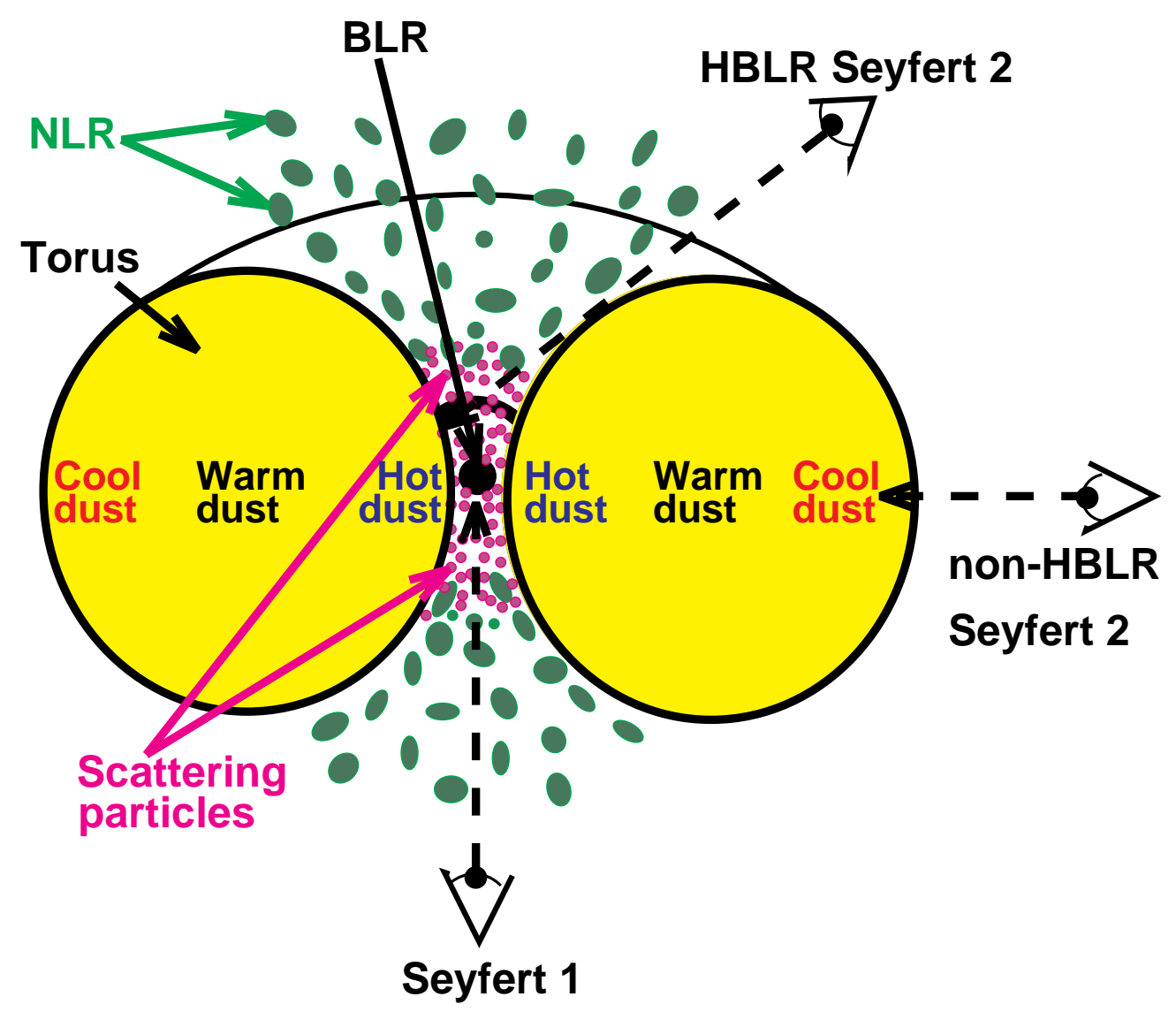

Figure 3-Schematic diagram (not to scale) of the central region of a Seyfert galaxy illustrating the effect of viewing angle on infrared colours and ability to detect the broad line region (BLR). We have indicated the BLR which has a typical size of $<1 \mathrm{pc}$, the narrow line region (NLR) clouds located $<1 \mathrm{kpc}$ from the BLR, and the scattering particles located $<300 \mathrm{pc}$ from the BLR. The temperature of the dust in the torus decreases radially outwards. The viewing angles producing a Seyfert 1, a Seyfert 2 with polarised broad lines and a Seyfert 2 without polarised broad lines are illustrated. An edge-on view will result in an optical spectrum which is characteristic of a Seyfert 2 with 'cool' infrared colours, and relatively large values of internal extinction, due to obscuration by the cool dust in the outer regions of the torus. A face-on viewing angle will provide a direct view of the BLR and of the hot dust at the inner edge of the torus resulting in a Seyfert 1 spectrum and 'hot' infrared colours. At intermediate viewing angles, the BLR is detected only by scattered light, the FIR colours have 'warm' dust temperatures, and the internal extinction to the NLR will be small relative to an edge-on orientation.

field. Such magnetic fields in AGN may be part of the internal structure of dense molecular torus clouds (Krolik \& Begelman 1988).

2.1.3 Results of spectropolarimetry survey. The most striking result of the Heisler, Lumsden \& Bailey survey is displayed in Figure 1. Those galaxies with detected polarised broad lines (represented by filled circles) have smaller values for the far-infrared (FIR) flux ratio $f_{60} / f_{25}$, and smaller Balmer decrements as measured by the ratio of the narrow lines, $\mathrm{H} \alpha / \mathrm{H} \beta$, compared to Seyferts for which polarised broad lines are not detected (represented by open squares). The segregation between Seyfert 2 galaxies with and without polarised broad lines is most obvious for the shorter wavelength ratios, $f_{60} / f_{25}$ and $f_{60} / f_{12}$ (Figure 2).

Is it possible that Seyferts with FIR flux ratios $f_{60} / f_{25}>4$ and lacking polarised broad lines in optical spectra are simply pure starburst galaxies without an AGN core? To address this question we have made use of data from the literature (Heisler,
Lumsden \& Bailey 1997; Roy et al. 1994; Norris et al. 1990) that was obtained using the ParkesTidbinbilla Interferometer (PTI) at $2 \cdot 3 \mathrm{GHz}$. The PTI is sensitive only to structures with brightness temperatures $>10^{5} \mathrm{~K}$, and sizes less than $0 \cdot 1^{\prime \prime}$, which corresponds to $<35 \mathrm{pc}$ at the mean redshift, $z=0 \cdot 018$, of our sample (assuming a Hubble constant $\left.H_{0}=75 \mathrm{~km} \mathrm{~s}^{-1} \mathrm{Mpc}^{-1}\right)$. Thus, the PTI is blind to extended star formation regions with typical brightness temperatures $<10^{4} \mathrm{~K}$ and ideal for detecting compact radio cores associated with AGN. There is no significant difference in the detection rate for Seyfert 2 galaxies with and without polarised broad lines, with $71 \%$ and $67 \%$ containing compact radio cores, respectively. These observed detection rates are consistent with that found for other samples of Seyfert 2 galaxies, and indicate that they are drawn from the same parent population of galaxies containing an active galactic nucleus. $\mathrm{X}$-ray emission is a powerful way for distinguishing AGN from starbursts, and it will soon be possible to 
undertake such a project with the Advanced X-ray Astrophysics Facility (AXAF).

The above results cannot be explained by a distance or luminosity effect. The redshift and FIR luminosity distributions are similar for the Seyferts with and without polarised broad lines. The Kolmogorov-Smirnov statistical test for the cumulative distributions of the redshift and FIR luminosity shows that we can reject the hypothesis that the two samples are different at the $96 \%$ and $98 \%$ confidence level, respectively.

Heisler, Lumsden \& Bailey (1997) interpret these results within the context of the unified model, with the assumption that the torus has a significant opacity, so that only the longer wavelengths $(>50 \mu \mathrm{m})$ could be assured of escaping directly. The inner side of the torus directly facing the active galactic nucleus has a high dust temperature $\left(T_{\text {dust }}>1000 \mathrm{~K}\right)$, contributing mostly at the shorter FIR wavelengths of 12 and $25 \mu \mathrm{m}$, and if we do not view the inner face of the torus almost directly, the 12 and $25 \mu \mathrm{m}$ radiation will be reprocessed to longer wavelengths, and the galaxy will have larger FIR flux ratios, $f_{60} / f_{25}$, compared to a face-on orientation. This is consistent with theoretical models (Pier \& Krolik 1993; Granato \& Danese 1994; Efstathiou \& RowanRobinson 1995) which indicate that an increase in the inclination of dust tori causes steepening of spectral indices at FIR wavelengths, and observational data (Giuricin, Mardirossian \& Mezzetti 1995; Maiolino et al. 1995; Spinoglio et al. 1995) which indicate that Seyfert 1 galaxies have smaller FIR flux ratios, $f_{25} / f_{12}$ and $f_{60} / f_{25}$, compared to Seyfert 2 galaxies. A major implication of these results is that the scattering particles producing the observed polarised broad lines must lie very close to or possibly within the plane of the torus. A schematic diagram illustrating how our results fit within the context of unification between Seyfert 1 and Seyfert 2 galaxies is displayed in Figure 3.

\subsection{Probing the Torus via Far-Infrared Colours}

The Heisler, Lumsden \& Bailey (1997) results show a clear progression of colour through Seyfert 2 galaxies with polarised broad lines to those in which polarised broad lines are not detected. This suggests a progression of increasing optical depths of dust. Recently, Dopita, et al. (1998) carried out an investigation to test the possibility that the IRAS flux ratios of Seyfert galaxies are attributed to extinction of an intrinsic Seyfert 1 spectrum (due to the emission by hot dust near its sublimation temperature) with optical depth which is variable from one object to another, and which tends to increase with viewing angle.

We began our investigation by plotting the positions of the Seyferts investigated by Heisler, Lumsden \& Bailey (1997) in the FIR flux ratio diagrams
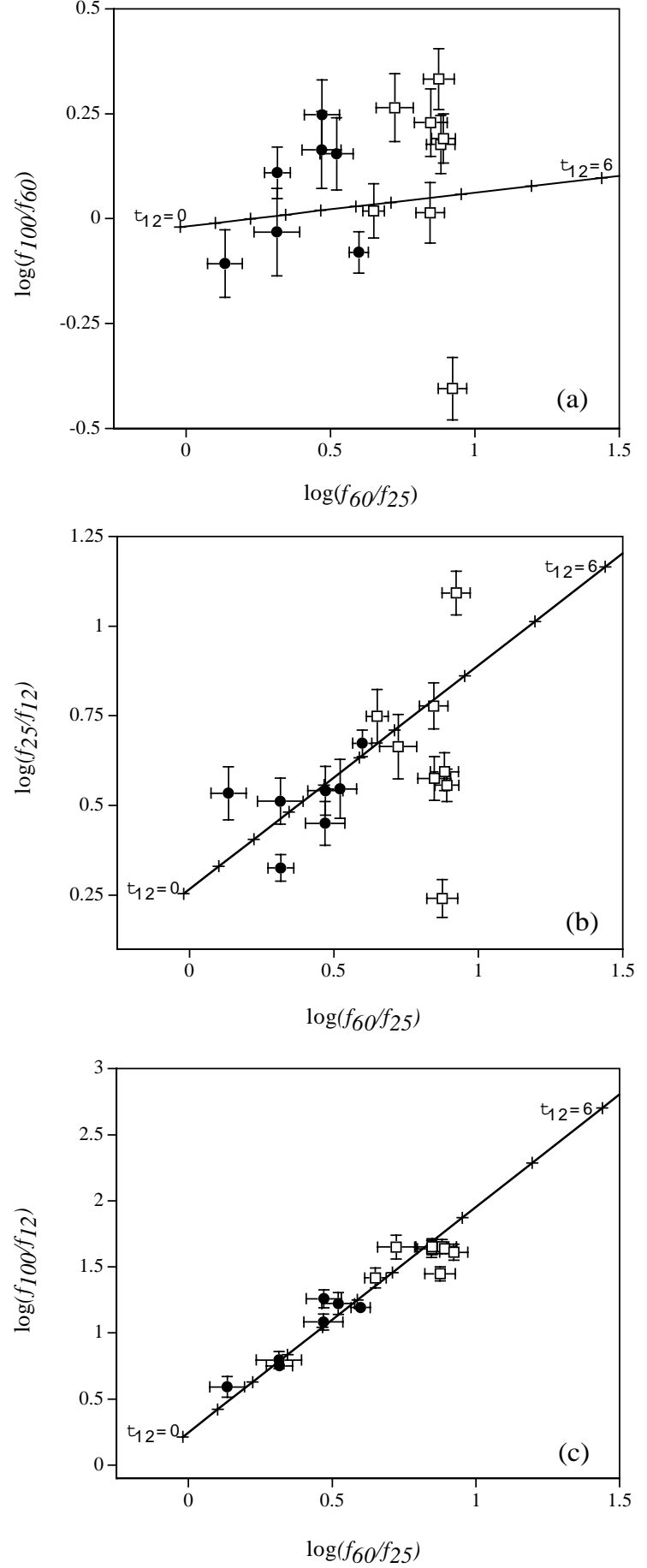

Figure 4-Far-infrared two-colour diagrams of (a) $\log \left(f_{60} / f_{25}\right)$ vs $\log \left(f_{100} / f_{60}\right)$, (b) $\log \left(f_{60} / f_{25}\right)$ vs $\log \left(f_{25} / f_{12}\right)$ and (c) $\log \left(f_{60} / f_{25}\right)$ vs $\log \left(f_{100} / f_{12}\right)$. The symbols have the same meaning as in Figure 1. The solid line represents the reddening line and the optical depths at $12 \mu \mathrm{m}$ are shown by tickmarks in the units $0,0 \cdot 5,1,1 \cdot 5,2,2 \cdot 5,3,3 \cdot 5,4$, 5,6 .

that are most commonly found in the literature: $\log \left(f_{60} / f_{25}\right) \log \left(f_{100} / f_{60}\right)$ and $\log \left(f_{60} / f_{25}\right)$ vs $\log \left(f_{25} / f_{12}\right)$ (Figures $4 \mathrm{a}$ and $4 \mathrm{~b}$, respectively). The solid line in these figures represents a theoretical reddening line computed using the infrared extinctions given by Dwek et al. (1997). The zero point 
for this reddening line was fixed using those Seyfert 1 galaxies with the smallest FIR flux ratios from the literature (Heisler \& Vader 1994; Rush, Malkan \& Spinoglio 1993). The assumption is that Seyfert 2 galaxies have the intrinsic colours of Seyfert 1 galaxies, reddened by various amounts of an obscuring screen of dust. The symbols have the same meaning as in Section $2 \cdot 1 \cdot 3$ where the Seyfert 2 with and without polarised broad lines are represented by filled circles and open squares, respectively. It is apparent that while many of the Seyferts with polarised broad lines lie within the warmer regions of the plots, there is a large scatter.

Stepping outside the boundary of the traditional IRAS flux ratio diagrams, we plotted the diagram of $\log \left(f_{60} / f_{25}\right)$ vs $\log \left(f_{100} / f_{12}\right)$. We obtained the surprising result that all of the Seyferts fall in a straight line along the reddening line, with the Seyferts observed to have polarised broad lines lying at the low extinction end of the plot (Figure 4c). As far as we are aware such a plot has not been previously published in the literature.

What causes such a tight correlation in this FIR flux ratio diagram? We know that $f_{60} / f_{25}$ discriminates between Seyfert 2 galaxies with and without polarised broad lines, presumably due to the dust torus being optically thick for wavelengths $<50 \mu \mathrm{m}$, indicating that this ratio is tied in with the temperature of the hot dust. The correlation between the ratio of $f_{100} / f_{12}$ and $f_{60} / f_{25}$ can be explained in terms of inclination of the torus to our line of sight as follows. When we view a Seyfert 2 galaxy nearly face-on and detect polarised broad lines, the FIR is dominated by emission from the hot dust near the nucleus, resulting in small values for $f_{60} / f_{25}$ and $f_{100} / f_{12}$. As the viewing angle is increased (referenced to the polar direction) the hot dust becomes obscured and the ratios reflect the cooler dust observed. As we progress to larger viewing angles (approaching edge-on), the FIR emission from the cooler regions of the outer tours and from the host galaxy itself start to dominate. This cool dust will eventually cause the ratio of $\log \left(f_{100} / f_{12}\right)$ to level off at a value $(\sim 1.6$ based on Figure 4c) determined by the ratio of the emissivity of silicates which dominate at $100 \mu \mathrm{m}$ (e.g. Puget et al. 1985), and the emissivity of poly-aromatic hydrocarbon (PAH) molecules which dominate at $12 \mu \mathrm{m}$. The effects of star formation on the FIR flux ratio plots are discussed further by Dopita et al. (1998).

The fact that the torus is optically thick for $\lambda>50 \mu \mathrm{m}$ will have potentially serious consequences for sample selection criteria of AGN. For example, consider samples of Seyferts chosen by their warm FIR colours, or small FIR flux ratios (e.g. de Grijp et al. 1985; Heisler 1991). These samples will have a bias towards warmer objects, and so, because most objects in it are close to the IRAS detection limit, the Seyfert 1 galaxies will tend to be slightly more distant than the Seyfert 2 galaxies. This will affect many statistical studies including number counts of Seyfert 1 and Seyfert 2 galaxies, and flux and luminosity distributions due to Malmquist bias. This now raises suspicion on the results of Roy et al. (1994) in which Seyfert 2 galaxies were found to have radio core detections more often than Seyfert 1 galaxies, as it may be attributed to the fact that the torus is optically thick at mid-infrared wavelengths (Norris, Heisler \& Roy 1998), and that Roy et al. chose Seyfert galaxies from the de Grijp et al. sample. As such, caution should be exercised when choosing samples of AGN.

\section{Evolution of AGN}

Although the simple dust obscuring torus accounts for the data remarkably well, it is not generally believed that AGN are static, non-evolving objects (see the review by Dopita 1997). During the period of time that a galaxy is undergoing Seyfert activity the presence of an obscuring torus plays a crucial role in determining whether we classify it as a Seyfert 1 or Seyfert 2. But the galaxies must evolve in the sense that there is a stage of time prior to the onset of nuclear activity and a stage after which nuclear activity has subsided. It is known that interactions between galaxies provide the means for funnelling gas towards the central regions of galaxies (e.g. Barnes \& Hernquist 1996; Bekki \& Noguchi 1994), and this may feed an existing massive black hole and/or create the onset of nuclear starbust activity which could eventually lead to the formation of an AGN (e.g. Planesas, Colina \& Perez-Olea 1997; Colina et al. 1997; Barnes \& Hernquist 1991; Norman \& Scoville 1988). Stellar bars, whose origin may be either internal or external, may also generate an inflow of gas towards the nucleus thereby leading to the onset of nuclear activity (e.g. Mulchaey \& Regan 1997; McLeod \& Rieke 1995).

\subsection{Compact Radio Cores in $A G N$}

Recently, Heisler et al. (1998) found that the IRASselected sample of $60 \mu \mathrm{m}$ Peakers have radio core luminosities which are intermediate between 'normal' Seyferts (i.e. FIR-selected Seyferts chosen without regard to FIR spectral energy distribution) and radio-loud ellipticals (Figure 5). The Seyfert $60 \mu \mathrm{m}$ Peakers $(60 \mathrm{PKs})$ follow the same relationship of 'core to total' flux as radio elliptical galaxies (Slee et al. 1994) (Figure 6). Thus the correlation between core and total power for radio ellipticals extends to Seyfert $60 \mathrm{PKs}$ with radio luminosities as low as $10^{20} \mathrm{~W} \mathrm{~Hz}{ }^{-1}$, and whose optical morphologies tend to be peculiar (i.e. tidal tails, rings, shells, double-nuclei) (Heisler \& Vader 1994), but whose near-infrared morphologies are well fitted by the 

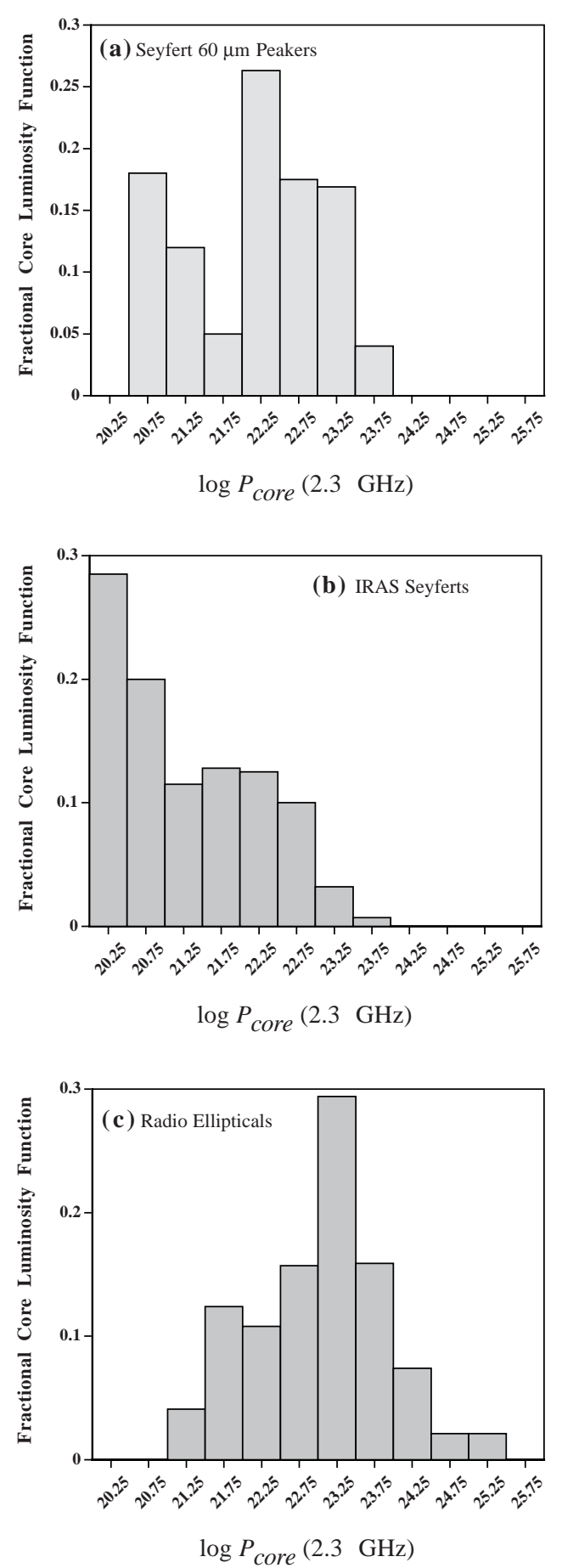

Figure 5-(a) Distribution function of radio cores for Seyfert 60 PKs. The median is $\log \left(P_{\text {core }}\right)=22 \cdot 1$. (b) Distribution function of radio cores for (1994). IRAS Seyfert galaxies without restriction to FIR spectral energy distribution from Roy et al. (1994). The median is $\log \left(P_{\text {core }}\right)=20 \cdot 9$. (c) Distribution function of radio cores for radio elliptical galaxies from Slee et al. (1994). The median is $\log \left(P_{\text {core }}\right)=23 \cdot 1$.

classical $r^{\frac{1}{4}}$ law for elliptical galaxies (Heisler, De Robertis \& Nadeau 1996). Thus, the compact radio cores in the centres of 60 PKs 'know' about the total radio emission in the galaxy. Compact radio cores are presumably associated with AGN, while the total radio emission is traditionally associated with star formation throughout the galaxy. However, it is noted that the radio emission in $60 \mathrm{PKs}$ is not like that of classical radio galaxies. It is unresolved on scales of $\sim$ kpc (Vader et al. 1993), and high resolution radio mapping is crucial for investigating the source of the total radio flux (i.e. radio jets or star formation).

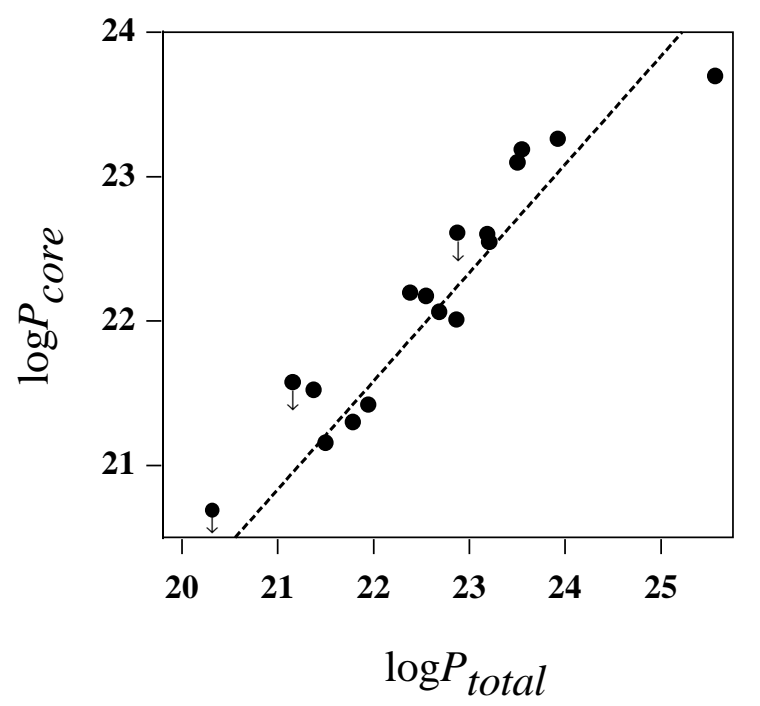

Figure 6-Relationship between core radio power and total radio power for Seyfert $60 \mu \mathrm{m}$ Peakers. The best fit line is given by $P_{\text {core }} \propto P_{\text {total }}^{0 \cdot 75}$.

\subsection{Far-Infrared-Radio Correlation}

There exists a tight correlation over a scale of several magnitudes between the radio and FIR fluxes for starburst and spiral galaxies (e.g. de Jong et al. 1985). Generally radio-quiet Seyfert galaxies show a larger scatter to this correlation (Norris, Allen \& Roche 1988), while Seyferts with strong radio cores do not fit the correlation (Sanders \& Mirabel 1985; Wilson 1988; Baum et al. 1993; Heisler et al. 1996; Roy \& Norris 1997). The evolutionary significance of these radio-excess galaxies (relative to the far-infraredradio correlation) has recently become an exciting controversial issue. It may be that these radio-excess objects are young radio galaxies in which the jet has yet to blast through the interstellar medium of the galaxy. Such a scenario has been proposed for the compact steep spectrum (CSS) and giga-hertz peaked spectrum (GPS) radio sources (e.g. Bicknell, Dopita \& O'Dea 1997). Recently, McGregor, Kewley \& van Breugel (1998) have identified a large sample of such intermediate radio-loud active galaxies, and find they have optical spectra with the signature of a post-starburst AGN. McGregor \& van Breugel are in the process of testing observationally whether there is a link between radio-excess galaxies and CSS sources, and thus whether they represent a transient phase in the evolution of AGN. 


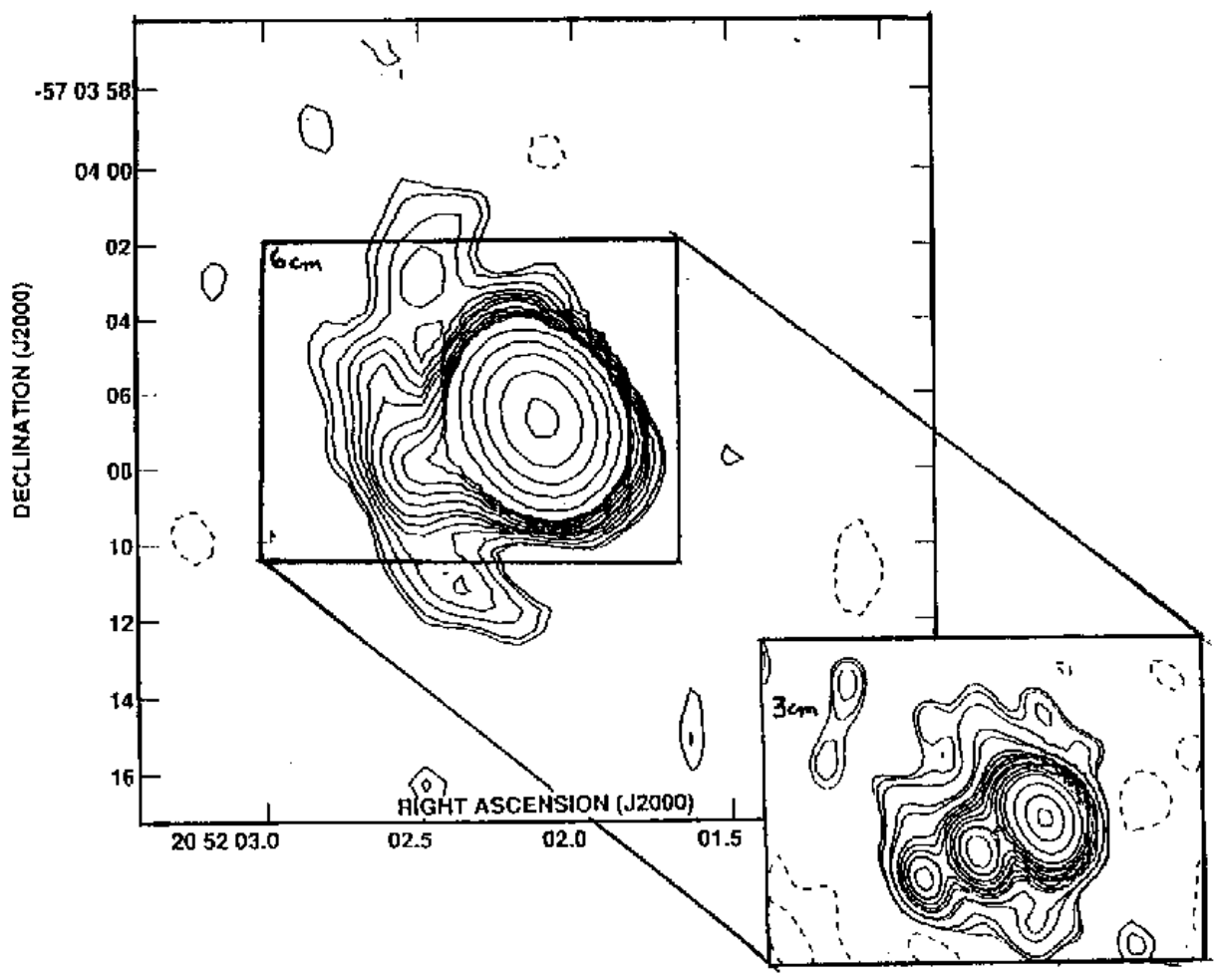

Figure 7-Image shows the $6 \mathrm{~cm}$ radio map of IC 5063 where the feature towards the east is believed to be a large-scale bow-shock, and the inset image shows the central region of IC 5063 at $3 \mathrm{~cm}$ where the linear triple radio source is clearly detected.

\subsection{Evolution of Radio Jets in AGN}

Bicknell et al. (1998) have recently examined the question of whether the narrow line regions in Seyferts can be powered by radio jets. This has important implications for the radio-excess Seyferts. The well known correlation between radio power and the luminosity of [OIII] $\lambda 5007$ in Seyfert galaxies (e.g. Whittle 1985) points to a connection between radioemitting and emission-line plasmas. It is well known that Seyfert galaxies containing luminous linear radio sources tend to have broader forbidden emission line widths indicating an additional acceleration mechanism which presumably involves the interaction of a jet with the circumnuclear interstellar medium (e.g. Whittle 1992). When this is combined with the observation that the radio axes in Seyferts correspond to the axes of the ionisation cones, it strongly suggests that the radio and optical emission are causally connected (e.g. Wilson \& Tsvetanov 1994). Based on this Bicknell et al. (1998) assert that the mechanical energy and momentum transported by the radio-emitting plasma is responsible for the excitation of some fraction of the narrow-line region. They calculate that in order for the NLR emission in Seyferts to be powered, at least in part, by radio jets, requires jet energy fluxes of order $10^{43-44} \mathrm{erg}$ $\mathrm{s}^{-1}$, and a smaller ratio of radio power to jet energy flux than is typically assumed for radio galaxies.
This lower value is attributed to the younger ages of Seyferts compared to radio galaxies. Many of the $60 \mathrm{PK}$ Seyferts lie in a region of the radio-[OIII] diagram which is intermediate between radio-quiet Seyferts and radio-loud ellipticals (Bicknell et al. 1998), which may be interpreted in terms of a transitional stage of evolution from radio-quiet to radio-loud due to the rapid growth of the central black hole as a result of a major merger. This interpretation is consistent with observational results (Heisler \& Vader 1994, 1995; Heisler, De Robertis \& Nadeau 1996; Heisler et al. 1998) that indicate Seyfert 60 PKs are intermediate between radio-quiet Seyferts and radio ellipticals and represent galaxies which have recently undergone a major merger event. High resolution radio mapping of objects such as 60 PKs will clarify the importance of jets in Seyfert galaxies. IC 5063 is a Seyfert 60 PK which is reasonably close and has been recently mapped in the radio, and I discuss it below.

3.3.1 The case of IC 5063. IC 5063 is classified as either a peculiar elliptical or $\mathrm{SO}$, and contains a series of dust lanes which strongly suggest a recent accretion event has occurred. The galaxy is a $60 \mu \mathrm{m}$ Peaker, and contains a hidden broad-line region discovered via spectropolarimetry (Inglis et al. 1993). The galaxy also exhibits a cross-shaped ionisation region, which Colina, Sparks \& Macchetto (1991) suggested is the edges of an ionisation cone 
illuminated by the central AGN. Figure 7 displays our discovery of a linear triple of radio emission at $3 \mathrm{~cm}$ and a possible large-scale bow-shock at $6 \mathrm{~cm}$ in IC 5063 (Bransford et al. 1998). The components of the triple are separated by approximately 3 arcsec corresponding to a linear scale of $500 \mathrm{pc}$. We interpret the linear triple source as the ejection of radio plasma from the nucleus of the galaxy. The central knot of the triple radio source is centred on the peak in the K-band continuum, suggesting that it is associated with the nucleus.

Triple radio sources, similar to that seen in IC5063, are commonly associated with Seyfert galaxies and are usually interpreted as being evidence for the ejection of plasma from the AGN. We have detected near-infrared line emission from the three knots [equivalent widths, for example in 1-0 S(1) molecular hydrogen, are 29 and 25 for the outer knots and 6 for the central knot]. This is consistent with the central radio knot being coincident with the brighter K-band continuum nucleus, and the strength of the emission from the outer radio knots supports the view that their associated plasma is interacting with the ISM surrounding the nucleus. The $\mathrm{H}_{2}$ emission is stronger in the outer knots than the nucleus, which is consistent with the idea that the outer knots have been ejected from the central source. Recently a strong blue-shifted $\left(-600 \mathrm{~km} \mathrm{~s}^{-1}\right.$ relative to the galaxy) HI absorption line has been seen against the radio sources by Morganti, Oosterloo \& Tsvetanov (1998). It was suggested by these authors that the absorption is evidence of outflow from the nucleus. We speculate that the HI absorption line is associated with entrained HI connected with the ejection event.

Because of the very dusty nature of this galaxy, we believe that a clear understanding of the dynamics of the inner regions can only come from IR spectroscopy. Planned observations with MPE 3D imaging spectrometer at the AAT will be used to provide a map of near-infrared emission lines across the radio sources and allow us (a) to resolve possible shock structures associated with the radio components, (b) to map the velocity field of the gas, and (c) to test the idea that the $21 \mathrm{~cm} \mathrm{HI}$ absorption results from gas entrained in the ejected radio plasma. With the velocity resolution provided by 3D it should be possible to determine whether the emission associated with the radio triple shows any evidence of outflow, and in particular whether the radio knots are associated with the $\mathrm{HI}$ absorption outflow.

\section{Conclusions}

With the use of optical spectropolarimetry on a welldefined sample of AGN, it has been demonstrated that the detectability of polarised broad lines depends upon FIR colours in a manner which can be understood within the context of the unified model for AGN. Emphasis is placed on the importance of sample selection when investigating properties of AGN, particularly in light of the evidence for a geometrically thick torus which is opaque to $\lambda<50 \mu \mathrm{m}$.

The importance of the evolution of AGN has been supported by observational evidence which strongly suggests that some types of AGN (in particular 'radio-excess' Seyferts and $60 \mu \mathrm{m}$ Peakers) represent the precursors to radio-loud AGN.

\section{Acknowledgments}

It is a pleasure to thank my colleagues for many enthusiastic and stimulating discussions about AGN and starburst activity. Thanks to Ray Norris, Mike Dopita, Stuart Lumsden, Jeremy Bailey, Phil Appleton, Geoff Bicknell, Peter McGregor, Mike De Robertis, Dave Jauncey and Mark Bransford. I am also grateful to my students, Tanya Hill and Lisa Kewley. I thank the referees for reading the manuscript and providing useful comments which have helped to clarify several points in the paper. I also thank Baerbel Koribalski, Lucyna KedzioraChudzer and Raffaella Morganti who have organised the AGN lunches - they have been an excellent medium for Australian astronomers studying AGN to get together and learn about research that is carried out by their colleagues. I am grateful to the TACs of the AAT, ATNF and MSSSO for the generous allocation of observing time that has allowed the successful completion of several projects that have been discussed in this review.

\section{References}

Antonucci, R. R. J. 1993, ARA\&A, 31, 473

Antonucci, R. R. J., \& Miller, J. S. 1985, ApJ, 297, 621

Barnes, J. E., \& Hernquist, L. E. 1991, ApJL, 370L, 65

Barnes, J. E., \& Hernquist, L. E. 1996, ApJ, 471, 115

Baum, S. A., O'Dea, C. P., Dallacassa, D., de Bruyn, A. G., \& Pedlar, A. 1993, ApJ, 419, 553

Bekki, K., \& Noguchi, M. 1994 A\&A, 290, 7

Bicknell, G. V., Dopita, M. A., \& O'Dea, C. P. O. 1997, ApJ, 485, 112

Bicknell, G. V., Dopita, M., Tsvetanov, Z., \& Sutherland, R. 1998, ApJ,495, 680

Bransford, M. A., Appleton, P. N., Heisler, C. A., Norris, R. P., \& Marston, A. P. 1998, ApJ, 497, 133

Colbert, E. J. M, Baum, S. A., Gallimore, J. F., O'Dea, C. P., Lehnert, M. D., Tsvetanov, Z., Mulchaey, J. S., \& Caganoff, S. 1996, ApJS, 105, 75

Colina, L., Garcia Vargas, M. L., Mass-Hesse, J. M., Alberdi, A., \& Drabbe, A. 1997, ApJ, 484L, 41

Colina, L., Sparks, W. D., \& Macchetto, F. 1991, ApJ, 382, 63

de Grijp, M. H. K., Miley, G. K., Lub, J., \& de Jong, T. 1985 Nature, 314, 240

de Jong, T., Klein, U., Wielebinski, R., \& Wunderlich, E. 1985 A\&A, 147, L6

Dopita, M. A. 1997 PASA, 14, 230 
Dopita, M. A., Heisler, C. A., Lumsden, S. L., \& Bailey, J. A. 1998, ApJ, 498, 1570

Dwek, E., et al. 1997, ApJ, 475, 565

Efstathiou, A., \& Rowan-Robinson, M. 1995 MNRAS, 273, 649

Gallimore, J. F., Baum, S. A., \& O'Dea, C. P. 1997 Nature, 388,852

Giuricin, G., Mardirossian, F., \& Mezzetti, M. 1995, ApJ, 446,550

Granato, G. L., \& Danese, L. 1994, MNRAS, 268, 235

Heisler, C. A. 1991, PhD Thesis, Yale University, New Haven

Heisler, C. A., \& Vader J. P. 1994, AJ, 107, 35

Heisler, C. A., \& Vader, J. P. 1995, AJ, 110, 87

Heisler, C. A., Lumsden, S. L., \& Bailey, J. A. 1997, Nature, 385,700

Heisler, C. A., Norris, R. P., Jauncey, D. L., Reynolds, J. E., King, E. A., \& Tzioumis, T. 1998, MNRAS, accepted

Heisler, C. A., De Robertis, M. M., \& Nadeau, D. 1996, MNRAS, 280, 579

Heisler, C. A., Norris, R. P., Jauncey, D. L., Reynolds, J. E., \& King, E. A. 1996, Fourth Asia Pacific Meeting and Workshop, ed. E. A. King (Sydney, ATNF), p. 166

Hines, D. C., \& Wills, B. J. 1993, ApJ, 415, 82

Inglis, M. D., Brindle, C., Hough, J. H., Young, S., Axon, D. J., Bailey, J. A., \& Ward, M. J. 1993, MNRAS, 263, 895

Khachikian, E. Y., \& Weedman, D. W. 1974, ApJ, 192, 581

Krolik, J. H., \& Begelman, M. C. 1988, ApJ, 329, 702

McGregor, P., Kewley, L., \& van Breugel, W. 1998, in preparation

McLeod, K., \& Rieke, G. 1995, ApJ, 464, 641

Maiolino, R., Ruiz, M., Rieke, G. H., \& Keller, L. D. 1995, ApJ, 446, 561

Miller, J. S., \& Goodrich, R. W. 1990, ApJ, 355, 456

Morganti, R., Oosterloo, T., \& Tsvetanov, Z. 1998, AJ, 115, 915

Mulchaey, J. S., \& Regan, M. W. 1997, ApJL, 482, 135L

Mulchaey, J. S., Wilson, A. S., \& Tsvetanov, Z. 1996a, ApJS, 102, 309

Mulchaey, J. S., Wilson, A. S., \& Tsvetanov, Z. 1996b, ApJ, 467,197
Norman, C., \& Scoville, N. 1988, ApJ, 332, 124

Norris, R. P., Allen, D. A., \& Roche, P. F. 1988, MNRAS, 234,773

Norris, R. P., Allen, D. A., Sramek, R. A., Kesteven M. J., \& Troup, E. R. 1990, ApJ, 359, 291

Norris, R. P., Heisler, C. A., \& Roy, A. L. 1998, in preparation.

Osterbrock, D. E. 1989, Astrophysics of Gaseous Nebulae and Active Galactic Nuclei (Mill Valley: University Science Books), pp. 308-81.

Peterson, B. M. 1993, PASP, 105, 247

Pier, E. A., \& Krolik, J. H. 1993, ApJ, 418, 673

Planesas, P. Colina, L., \& Perez-Olea, D. 1997, A\&A, 325, 81

Pogge, R. W. 1989, ApJ, 345, 730

Puget, J. L., Leger, A., \& Boulanger, F. 1985, A\&A, 142, L19

Roy, A. L., \& Norris, R. P. 1997, MNRAS, 289, 824

Roy, A. L., Norris, R. P., Kesteven, M. J., Troup, E. R., \& Reynolds, J. E. 1994, ApJ, 432, 496

Rush, B., Malkan, M. A., \& Spinoglio, L. 1993, ApJS, 89, 1

Sanders, D. B., \& Mirabel, I. F. 1985, ApJL, 298, L31

Seyfert, C. K. 1943, ApJ, 97, 28

Simpson, C., Wilson, A. S., Bower, G., Heckman, T., Krolik, J. H., \& Miley, G. K. 1997, ApJ, 474, 121

Slee, O. B., Sadler, E. M., Reynolds, J. E., \& Ekers, R. D. 1994, MNRAS, 269, 928

Spinoglio, L., Malkan, M. A., Rush, B., Carrasco, L., \& Recillas-Cruz, E. 1995, ApJ, 453, 616

Tran, H., Miller, J. S., \& Kay, L. 1992, ApJ, 397, 452

Urry, C. M., \& Padovani, P., 1995, PASP, 107, 803

Vader, J. P., Frogel, J. J., Terndrup, D. M., \& Heisler, C. A. 1993, AJ, 106, 1743

Webb, W., Malkan, M., Schmidt, G., \& Impey, C. 1993, ApJ, 419, 494

Whittle, M. 1985, MNRAS, 213,

Whittle, M. 1992, ApJ, 387, 121

Wilson, A. S. 1988, A\&A, 206, 41

Wilson, A. S., \& Tsvetanov, Z. I. 1994, AJ, 107, 1227

Young, S., Hough, J. H., Efstathiou, A., Wills, B. J., Bailey, J. A., Ward, M. J., \& Axon, D. J. 1996, MNRAS, 281, 1206 